\title{
Aneurysm Surgery in Asia
}

-Japanese Situation-

\author{
Yuichiro TANAKA, Shigeaki KOBAYASHI, Michihiko OSAWA, \\ Kazuhiko KYOSHima, Hiroshi OKUDERA, Tsuyoshi TADA, \\ Kazuo KitazaWA, and Susumu OIKaWA
}

Department of Neurosurgery, Shinshu University School of Medicine, Matsumoto, Nagano

\begin{abstract}
This report is based on the results of the questionnaires conducted for the Japan Stroke Surgery Meeting 1996 (President: S. Kobayashi). The questionnaires were sent to all neurosurgical training institutions approved by The Japan Neurosurgical Society, numbering 959. The response rate was $54.2 \%$ (520 institutions). All statistics dealt with cases from January 1995 to December 1995. The total number of aneurysm surgery performed was 13,166 . The average operative case number per institution during the year was 25 . About $20 \%$ of the institutions exceeded 35 cases. Of all operative cases, $76.3 \%$ were ruptured aneurysms and $23.7 \%$ were nonruptured. Giant aneurysms were $2.9 \%$; dissecting aneurysms $2.4 \%$. Surgical procedures performed were clipping in $90.3 \%$, wrapping in $5.2 \%$, proximal ligation $1.6 \%$, and bypass in $0.7 \%$. Intravascular surgery was performed for $2.3 \%$ of the cases. Sugita clips were mainly used in $80.5 \%$ of the institutions, Yasargil clips in $6.6 \%$, and both in $12.9 \%$. For anterior communicating artery aneurysms pterional approach was mainly used in $81.0 \%$ of the institutions, interhemispheric in $7.2 \%$, and both in $11.7 \%$. For basilar terminal aneurysms, pterional approach was mainly used in $88.3 \%$ of the institutions, subtemporal approach in $6.6 \%$, and both in $5.1 \%$. The nonoperative cases included $24.9 \%$ of the ruptured aneurysms, $31.7 \%$ of the unruptured aneurysms, $38.4 \%$ of the giant aneurysms, and $52.1 \%$ of the dissecting aneurysms. The above statistics suggest that aneurysms are treated in Japan in most training institutions and that open surgical treatment is still the main procedure of choice.
\end{abstract}

Key words: aneurysm surgery, statistics, aneurysm clips, surgical approach

\section{Introduction}

Treatment of intracranial aneurysms has undergone various changes due to the introduction of new diagnostic techniques and surgical instruments. In recent years, in particular, intravascular surgery has made another therapeutic challenge for aneurysms. An increasing number of unruptured intracranial aneurysms are detected by noninvasive diagnostic tools such as magnetic resonance angiography and three-dimensional computed tomography scanning. With these new innovations, forming more effective treatment regimens is needed for the treatment. In order to do this, we considered it important to grasp the current status of treatment of intracranial aneurysms. This report is based on the results of the questionnaires conducted for the Japan Stroke Surgery Meeting in 1996 (President: S. Kobayashi) (some of the results were reported previously, ${ }^{3 \text { l) }}$ data in this paper are cited with permission).

\section{Methods}

The questionnaires were sent to all neurosurgical training institutions approved by The Japan Neurosurgical Society, numbering 959 as of 1997 . The questionnaires were consisted of 17 items concerning operative and nonoperative cases treated at these institutions between January and December of 1995 (Table 1). ${ }^{3)}$ The institutions where the questionnaires were sent were classified into Group I: national university hospitals (prefectural or city university hospitals included), Group II: private university hospitals, Group III: other category A (independent training) hospitals, and Group IV: category C (affiliated training) hospitals.

\section{Results}

Effective response was obtained from 520 of the 959 institutions (response rate $54.2 \%$ ). 13,166 operative 
Table 1 Questionnaire

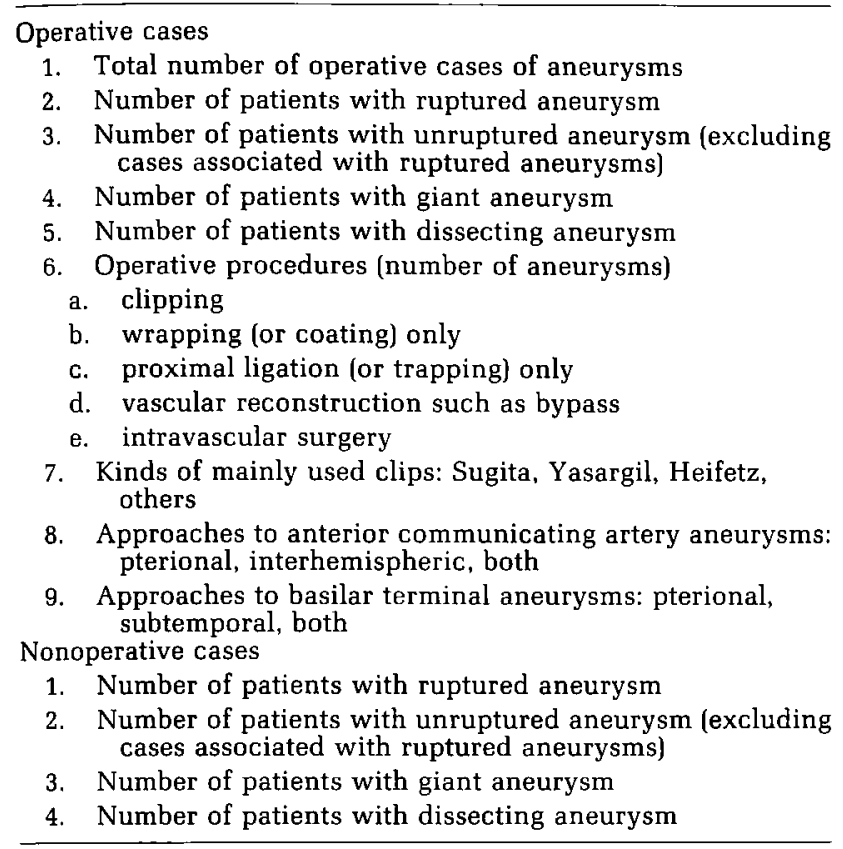

Reproduced with permission from Kobayashi et al: $\mathrm{Re}$ cent treatment of cerebral aneurysm in Japan. Surgery for Cerebral Stroke 25: 91-96, 1997.3)

patients and 4788 nonoperative patients were submitted for analysis. $76.3 \%$ of the patients who had undergone surgical procedures had ruptured aneurysms while $23.7 \%$ nonruptured aneurysms (Fig. 1). ${ }^{3}$ Of the nonoperative cases, $69.7 \%$ were for ruptured aneurysms while $30.3 \%$ unruptured aneurysms. Of all the ruptured aneurysms, $75.1 \%$ were operated and $24.9 \%$ not operated. Of all the nonruptured aneurysms, $68.3 \%$ were operated and $31.7 \%$ not operated.

The average operated cases per institution was 25 during the one year period. Figure 2 shows the numbers of the operative cases at the institutions in the increasing order. ${ }^{3)}$ The top ten institutions included five private hospitals and two public hospitals in local cities (both Group III), and two private university hospitals (Group II) and one public hospital (Group III) in the metropolitan area. Six institutions operated on more than 100 operative cases a year, where unruptured aneurysm cases occupied $43 \%$ on average. 437 of the 520 institutions (84\%) operated on more than 10 cases a year; in 53 of those institutions unruptured aneurysm numbered more than $50 \%$ of the entire operative cases.

The incidence of giant aneurysms was $2.9 \%$ and that of dissecting aneurysms $2.4 \%$. $61.6 \%$ of the giant aneurysms underwent surgery, while $38.4 \%$ did not. $47.9 \%$ of the dissecting aneurysms had surgery

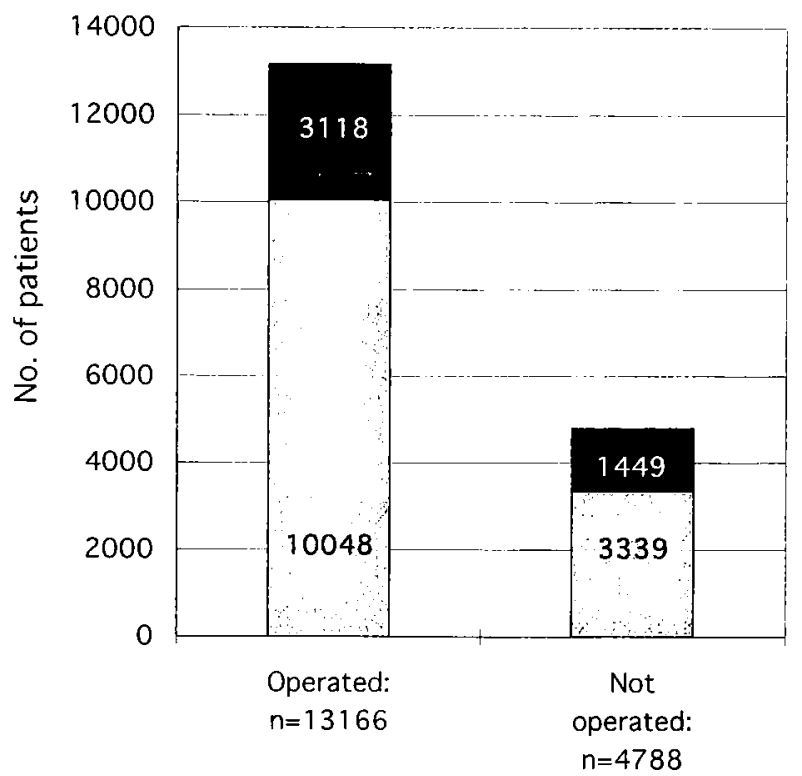

Fig. 1 Each bar showing the total number of the patients with cerebral aneurysms with or without operation in 520 hospitals in Japan. The hatched column indicates unruptured aneurysms and the shaded column indicates ruptured ones. Reproduced with permission from Kobayashi et al: Recent treatment of cerebral aneurysm in Japan. Surgery for Cerebral Stroke 25: 91-96, 1997.

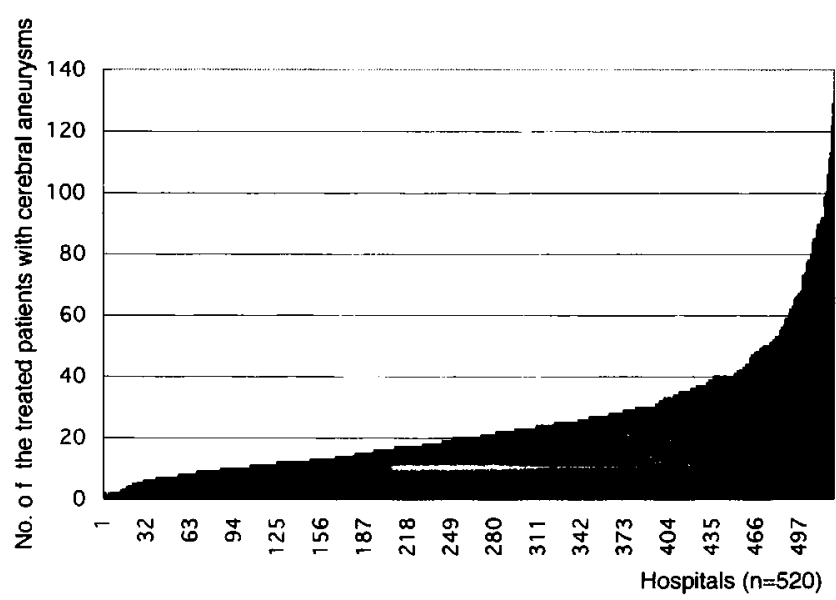

Fig. 2 A graph showing the number of patients with a cerebral aneurysm in $\mathbf{5 2 0}$ hospitals in 1995. Reproduced with permission from Kobayashi et al: Recent treatment of cerebral aneurysm in Japan. Surgery for Cerebral Stroke 25: 91-96, 1997.3) 
and $52.1 \%$ did not. Giant aneurysms were operated on at 159 institutions (30.6\%) and the dissecting aneurysms at 175 institutions (33.7\%).

Surgical procedures performed were clipping in $90.3 \%$, wrapping or coating in $5.2 \%$, intravascular surgery in $2.3 \%$, and proximal ligation or trapping in $1.6 \%$; bypass-combined procedures in $0.7 \%$ of the cases (64 institutions). Intravascular procedures were performed in 104 institutions (20\%); however, in 74 institutions, the number of intravascular procedures were less than two cases a year for aneurysm. Response to clip selection was from $513(98.7 \%)$ institutions. Sugita clips were mainly used in $80.5 \%$, Yasargil in $6.6 \%$, and both in $12.9 \%$ of the institutions.

For anterior communicating artery aneurysms, 511 of the 520 institutions $(98.3 \%)$ responded. Mainly pterional approach was used in $81.0 \%$ of the institutions, interhemispheric approach in $7.2 \%$, and both approaches in $11.7 \%$. For basilar terminal aneurysms, 316 institutions responded (60.8\%). Pterional approach was mainly used in $88.3 \%$ of the

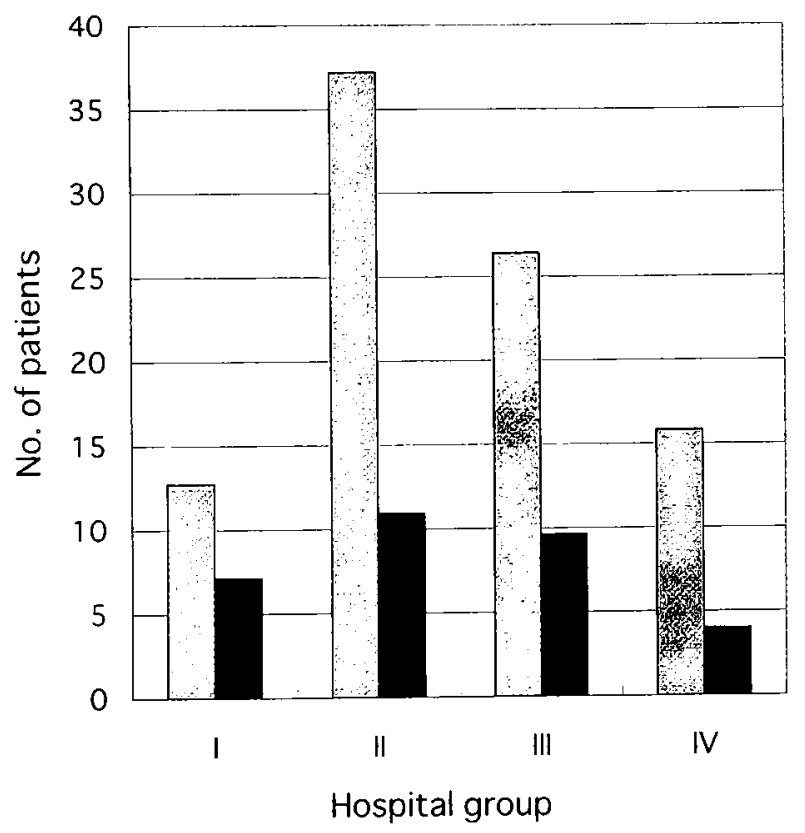

Fig. 3 Each bar showing the averaged number of the ruptured (shaded column) and unruptured aneurysms (hatched column) which were operated on in Group I to IV hospitals. Group I: national university hospitals, II: private university hospitals, III: other category A hospitals, IV: category C hospitals. Reproduced with permission from Kobayashi et al: Recent treatment of cerebral aneurysm in Japan. Surgery for Cerebral Stroke 25: 91-96, 1997.. institutions, subtemporal approach in $6.6 \%$, and both in $5.1 \%$.

Of all the respondents, 45 institutions belonged to Group I, 24 to Group II, 117 to Group III, and 334 to Group IV. The average operative cases per institution is $19.8,48.1,36.0$, and 19.8 for Group I, II, III, and IV institutions, respectively. Unruptured aneurysms occupied $35.7 \%, 22.8 \%, 26.8 \%$, and $20.5 \%$ of all operated cases for Group I to IV institutions, respectively, showing that national university hospitals operate on a greater number of unruptured aneurysms than other groups (Fig. 3). ${ }^{3]}$

\section{Discussion}

Subarachnoid hemorrhage caused by rupture of intracranial aneurysms is known to occur in about 12 per 100,000 population. Suppose the population of Japan is $12,600,000$, the incidence of subarachnoid hemorrhage would be about 13,000 per year. The statistics of the present questionnaire show that the number of the patients with subarachnoid hemorrhage was 15,000 in the year studied. Although the response rate was $54 \%$, the responding institutions all together seem to have operated on the majority of the cases. Knowing that the number of board certified neurosurgeons in Japan was 3067 and that of all vascular surgeries performed was 23,652 in 1995 , the average number of vascular surgical cases per one neurosurgeon was about eight (data provided by The Japan Neurosurgical Society); the average number of aneurysm surgery cases would be about four to five.

As to the distribution of the cases, the operative cases of aneurysm in Japan seem to be distributed widely among institutions, while a large number of cases are operated on in some hospitals. Cases are concentrated in large referral center hospitals outside the metropolis, and in private university hospitals in the metropolitan area. On the other hand, cases are scattered in smaller scaled institutions as the number of institutions and board certified neurosurgeons have increased. Surgical indications for unruptured aneurysms differ according to different hospitals but on average their number was one fourth of the total operative cases.

From the point of view of difference in institution categories, generally Group II (private university) hospitals operate more than Group I (national, prefectural, or city university) hospitals. Group I hospitals operate on more unruptured and giant aneurysms than hospitals of other groups. In the present study, questions were not asked about the timing of surgery. Generally in Japan ruptured aneurysms are operated on in acute stage unless sur- 
gical grades are poor. Emergency medical care system is generally well developed in private university hospitals than in national hospitals. And therefore, the former attract more acute patients.

When patients with ruptured aneurysm were admitted to the hospitals, about one fourth of them were not operated on, which corresponds to the reported ratio of $20 \%$ to $33 \%$ of inoperable ruptured aneurysm cases. ${ }^{2,45)}$ It is to be noted that despite the development of improved treatment of acute subarachnoid hemorrhage, the ratio of operability has not changed in the past decade.

As to selection of methods for surgical treatment of aneurysms, despite recent introduction of intravascular surgery, clipping was employed for $90 \%$ of the cases, indicating that this method is still the main procedure of choice. Wrapping and coating were used for about $5 \%$ of the cases, which is not a negligible percentage. Wrapping materials were not uniform and differed according to different institutions.

There have been much discussion in Japan as to the approach to the anterior communicating artery aneurysm; however, the present survey has found that the pterional approach is employed significantly more than the interhemispheric approach. The institutions employing the latter approach numbered 37 which is less than $10 \%$ of all institutions; 60 institutions selected either of the two for individual cases.

As to the approach to the basilar terminal aneurysms (basilar bifurcation and basilar-superior cerebellar artery aneurysms), the pterional approach $^{6,7)}$ is predominantly used in Japan, contrasting to the report of the overwhelming number of cases operated on via the subtemporal approach by Drake. ${ }^{1)}$ It is to be noted that $39 \%$ of the institutions did not operate on basilar terminal aneurysms, indicat- ing the rare occurrence of this type of aneurysm and probably technical difficulty in clipping these aneurysms.

\section{References}

1) Drake CG: Treatment of aneurysms of the posterior cranial fossa. Progress in Neurological Surgery 9: 122144,1978

2) Hata $S$, Oh Dal S, Ishi S: Cooperative study on ruptured aneurysm in Japanese neurosurgical clinics. Neurol Med Chir (Tokyo) 23: 30-40, 1983

3) Kobayashi S, Tanaka Y, Osawa M, Kyoshima K, Tsuji T, Okudera K, Tada T, Miyashita T: [Recent treatment of cerebral aneurysm in Japan]. Surgery for Cerebral Stroke 25: 91-96, 1997 (Jpn, with Eng abstract)

4) Locksley HB: Report of the cooperative study of intracranial aneurysms and subarachnoid hemorrhage: section 5, part 1. Natural history of subarachnoid hemorrhage, intracranial aneurysms and arteriovenous malformations based on 6368 cases in the cooperative study. I Neurosurg 26: 413-416, 1966

5) Nishioka H: Report on the cooperative study of intracranial aneurysms and subarachnoid hemorrhage, section VII, part I: Evaluation of the conservative management of ruptured intracranial aneurysms. J Neurosurg 25: 574-600, 1966

6) Sugita K, Kobayashi S, Shintani A, Mutsuga N: Microneurosurgery for aneurysms of the basilar artery. J Neurosurg 51: 615-620, 1979

7) Yasargil MG, Antic J, Laciga R, Jain KK, Hodosh RM, Smith RD: Microsurgical pterional approach to aneurysms of the basilar bifurcation. Surg Neurol 6: $83-91,1976$

Address reprint requests to: S. Kobayashi, M.D., Department of Neurosurgery, Shinshu University School of Medicine, 3-1-1 Asahi, Matsumoto, Nagano 3908621 , Japan. 